\title{
Impact of insulin pump use on disease control and quality of life in type I diabetes patients
}

\begin{abstract}
Aim: The aim of the study is to objectively assess the benefits that patients gain by switching from conventional insulin injections to insulin pump therapy.

Introduction: An Insulin pump is a device used to treat diabetes and by infusing the insulin through a subcutaneous needle. It has become increasingly popular over the last several decades. Its mode of action is the closest mimicker to the pancreas and in theory it should properly manage blood sugar. However its impact on diabetes control was found to be modest and the remarkable improvement in glycemic control was demonstrated in observational studies and could not be confirmed by randomized controlled trails.

Methods: Our study is an observational cross sectional study done in a single center. Forty patients with type I diabetes on insulin pump therapy were retrospectively assessed and compared with a matched control group that received multiple daily insulin injections. Biochemical data were collected including hemoglobin A1C (HbA1c), and all patients were interviewed and asked to complete a validated questionnaire to assess the quality of life.

Results: Compared with the control group, patients on insulin pump therapy showed significantly lower $\mathrm{HbA} 1 \mathrm{c}$ levels, with a mean of 1.8 , had fewer hypoglycemic episodes, and had higher scores on the quality of life questionnaire.

Conclusion: In our center, the insulin pump therapy was shown to remarkably improve the diabetes control and quality of life in type I diabetes to a greater extent than what has been reported in many observational studies.
\end{abstract}

Keywords: diabetes mellitus, hypoglycemia, hemoglobin A1C, insulin pump, quality of life
Volume 6 Issue 2 - 2018

\author{
Mohammed Alqambar,Ahmed ALOthman, \\ Mazen Elyahia, Mutlaq ALSubaie, Wadee \\ ELhakimi, Abdulaziz Alwosaibi, Jamal Alsaeed, \\ Ibrahim Alkadhim, Nada Alhabib, Maryam \\ Alqarrous, Mohammed Sammak, Fadhel \\ Alhaddad \\ Endocrinology Section, Department of Medicine, King Fahad \\ specialist Hospital, Dammam, Saudi Arabia
}

\begin{abstract}
Correspondence: Mohammed Alqambar, Endocrinology Section, Department of Medicine, King Fahad specialist Hospital, Po Box 15215 Dammam 3 I444 Saudi Arabia,Tel +966549I3055I, Email ALQAMBARM@YAHOO.COM
\end{abstract}

Received: February 25, 2017| Published: April II, 2018

\section{Introduction}

An Insulin pump is a device that is used to treat diabetes by infusing insulin into the subcutaneous space through a needle that is usually kept in place for three days. The insulin is infused by the pump in two phases: continuous infusion, also called the basal insulin, and bolus form, or prandial insulin injected with each meal. ${ }^{1}$ This therapy has become increasingly popular over the last several decades. Theoretically, the concept of insulin secretion by the pump is the closest mimicker of pancreatic function. However, its impact on diabetes control, as measured by hemoglobin A1C (HbAlc) was found to be modest $\mathrm{t}^{2-5}$ and was only proven to be remarkable by observational studies; randomized controlled trials failed to confirm substantial improvement in HbAlc. ${ }^{3}$ Insulin pumps have been shown to improve patient's quality of life and decrease the rate of hypoglycemic episodes. ${ }^{7}$ In this study our goal is to assess the impact of insulin pump therapy on our patients.

Our research question is how much benefit and what benefits do type I diabetes patient's gain by switching them from the conventional subcutaneous insulin injections to insulin pump therapy.

\section{Primary objectives}

To evaluate the impact of insulin pump therapy on quality of life and glycemic control.

\section{Secondary objectives}

To assess the benefit of insulin pump therapy on the rate of hypoglycemia, total insulin dose per day, frequency of Diabetes Ketoacidosis (DKA), and number of hospital admissions.

\section{Methods}

\section{Study design}

By using a cross sectional design, among eighty patients on insulin pump therapy treated between 2008 and 2014, forty of them full filled our inclusion criteria (pump group) and they were assessed and compared with forty patients on insulin injections (control group) who were matched based on age, duration of diabetes, number of episodes of hypoglycemia, number of hospitalizations, frequency of developing diabetes ketoacidosis, body mass index, HBA1c and other factors at the time of starting the insulin pump therapy, as shown in Table 1. The mean duration of pump use was 3.5 years.

The study was approved by the hospital ethical board committee. All patients were treated at King Fahad Specialist Hospital, which is a tertiary governmental hospital in the city of Dammam in the Eastern province of Saudi Arabia. We used the following inclusion criteria to enroll patients in the study: presence of type I diabetes, age between 18 and 60 years, and receiving insulin pump therapy for at least six months and the device must have been installed at our institution. 
Patients were excluded if they lacked laboratory results for at least six months or if they had blood disorders or stage four renal failures

which could make the HbA1c measurement unreliable (Table 1).

Table I Baseline characteristics of pump group (at the time of initiating the insulin pump) and control group

\begin{tabular}{llll}
\hline & $\begin{array}{l}\text { Pump Group } \\
(\mathbf{n}=\mathbf{4 0})\end{array}$ & $\begin{array}{l}\text { Control group (Insulin } \\
\text { Injections) }\end{array}$ & P-value \\
\hline Mean age (years) & 28.08 & 25 & 0.072 \\
Gender (\%) & M:30\%, F:70\% & M:40\%, F: 60\% & 0.35 \\
Mean BMI & 23 & 24.8 & 0.25 \\
Mean HbAIC (\%) & 9.1525 & 8.945 & 0.59 \\
Mean duration of diabetes (years) & 14.8 & 12.9 & 0.25 \\
Insulin dose (units/day) & 67.85 & 75.8 & 0.239 \\
Family history of type I diabetes (\%) & $42.50 \%$ & $50 \%$ & 0.5 \\
DKA/6 M & 1.2 & 0.63 & 0.16 \\
Hypoglycemia episodes/week & 3.175 & 2.05 & 0.014 \\
Hypothyroidism (\%) & $4.08 \%$ & $4.66 \%$ & 0.868 \\
TTG Positive (\%) & $18.80 \%$ & $4.80 \%$ & 0.175 \\
GFR (mean) & 129.06 & 131.11 & 0.466 \\
ACR (mean) & 3.2 & 11.09 & 0.216 \\
\hline
\end{tabular}

Abbreviations: BMI, Body mass index, $\mathrm{kg} / \mathrm{m}^{2}$; HbAIC, hemoglobin AIC; DKA, diabetes ketoacidosis; SMBG, self-monitoring of blood sugar; 6 M, mean number per six months; day, mean number per day; week, mean number per week;TTG, tissue transglutaminase antibodies; GFR, glomerular filtration rate; $\mathrm{n} 98$ - I56 ml/ min;ACR, albumin to creatinin ration; $\mathrm{n}, 0-2.5 \mathrm{mg} / \mathrm{mmol}$.

\section{Data}

All patients were interviewed, consented, and asked about the number of hypoglycemic episodes that they have per week and number self-blood sugar monitoring (SBSM) per day. Patients were asked to complete a questionnaire to assess their disease insight, quality of life and satisfaction with therapy. The questionnaire was validated for assessing quality of life in patients with diabetes. ${ }^{6}$ The other required information was obtained from the patients charts. All clinical data including laboratory test results were consolidated in a data base file by the research team. Hypoglycemia was defined as any neurogenic or neuroglycopenic symptom with a documented blood sugar level below $3.5 \mathrm{mmol} /$ liter. Each patient was asked how many times per week he experiences a hypoglycemic episode. Severe hypoglycemia, was defined as neuroglycopenic symptoms with a documented blood glucose below $3.5 \mathrm{mmol} / \mathrm{liter}$ that required help from another person to resolve. Each patient was asked to estimate the number of sever hypoglycemic episodes experienced per month. We chose a longer period to capture larger number of episodes, as required for statistical analysis.

All patients in the pump group were using the Medtronic Paradigm 754 VEO insulin pump, and the insulin used for the pump was aspart which is an ultra-short acting insulin manufactured by Novo Nordisk, Bagsvaerd, Denmark. The insulin pumps were provided to patients for free. The control group was managed by conventional multiple insulin injections. Almost all of them used insulin aspart as prandial insulin and glargine (product of Sanofi, Paris, France) or detemir, manufactured by Nov Nordisk as basal insulin. All forms of insulin were used through a pen.

The laboratory tests in the study were done using the following assays and methods, HbAlc was measured by high-performance liquid chromatography using Bio-Rad VARIANT II TURBO 2.0 and the results were calibrated to DCCT numbers. TSH (Thyroid stimulating hormone) was measured by Abbott immunoassay Analyzer Si2000 (Abbot Diagnostics, Santa Clara, California, USA). Tissue transglutaminase (TTG) antibodies were measured using ELISA immunoassay (INOVA Diagnostics, San Diego, California, USA). Albumin to creatinine ratio (ACR) was measured with Dimension ${ }^{\circledR}$ RxL Max ${ }^{\circledR}$ clinical chemistry system using microalbumin assay. Glomerular filtration rate (GFR) was measured by Dimension ${ }^{\circledR}$ RxL Max ${ }^{\circledR}$ clinical chemistry system using Jaffee creatinine colorimetric assay.

\section{Pump protocol}

In our institution we use the American Association of clinical Endocrinology/American College of Endocrinology (AACE/ACE) guidelines to select appropriate candidates for insulin pump therapy. ${ }^{7}$ Based on the most recent AACE/ACE guidelines (2014), to be eligible for insulin pump therapy, patient should fulfill the following criteria: be clear from any psychiatric or behavioral disorders, be motivated to improve his blood sugar, be capable of using pump technology, self-monitor blood sugar (defined as at least four blood sugar measurements per day), be adherent to insulin therapy and be consistent in attending endocrinology and education clinics. When a patient is deemed eligible, he is evaluated by the diabetic educator who trains him thoroughly on the principals and details of pump therapy. After having several sessions he takes an exam to evaluate comprehension. The patient also attends several sessions with dietitian to learn carbohydrate counting. The whole process may take four to six weeks depending on patient comprehension. Finally, pump is initiated in an outpatient setting with very close follow up by our educators. The patient and his family receive clear instructions about the pump setting and how frequently the blood sugar should be measured. 
A hotline is also provided for any questions that may arise. The patient is instructed to present to the emergency room if needed. A follow up plan is arranged every 3 days with a diabetic educator and every month with endocrinology clinic until the pump regimen is stabilized.

\section{Statistical analysis}

The collected data were analyzed using Statistical Package for Social Sciences (SPSS) and checked for reliability and validity. Frequency tables were drawn to explore the outcome. Frequency tables for all variables were designed to show percentages, measures of central tendency, dispersion and graphics. Contingency tables, t-test, correlation co-efficient and regression analysis were used to compare the study group and the control group at a $95 \%$ significance level $(\mathrm{p}=<0.05)$

\section{Results}

Compared with control group, insulin pump users had statistically significant lower HbAlc levels, with a mean of 1.8. They also had better quality of life, a smaller percentage of them considered the disease to be burdensome ( $41.7 \%$ vs. $75 \%)$ and they had higher scores for treatment satisfaction. For the secondary endpoints, the pump group had a statistically lower risk of overall hypoglycemia (mean number of episodes of 1.08 vs. 2.05 per week), smaller daily insulin doses and more SMBG. There was no significant difference between the two groups in severe hypoglycemia, frequency of DKA and admission, as shown in Table 2.

Table 2 Final results of comparison between the pump and control groups

\begin{tabular}{llll}
\hline & $\begin{array}{l}\text { Pump group } \\
(\mathbf{n}=\mathbf{4 0})\end{array}$ & $\begin{array}{l}\text { Control } \\
\text { group } \\
(\mathbf{n}=\mathbf{4 0})\end{array}$ & P-value \\
\hline Mean HgAlc (\%) & 7.288 & 8.945 & 0.0001 \\
$\begin{array}{l}\text { Insulin dose (units/day) } \\
\text { Hypoglycemia/week }\end{array}$ & 42.13 & 75.8 & 0.0001 \\
Severe hypoglycemia/M & 1.08 & 2.05 & 0.011 \\
DKA/6 M & 0.15 & 0.6 & 0.069 \\
Admission/6 M & 0.48 & 0.63 & 0.688 \\
$\begin{array}{l}\text { Number of SMBG/day } \\
\text { Satisfaction with treatment } \\
\text { (\%) }\end{array}$ & 0.38 & 0.65 & 0.421 \\
$\begin{array}{l}\text { High Burden of the disease } \\
\text { (\%) }\end{array}$ & 51.95 & 2.36 & 0.04 \\
\hline
\end{tabular}

Abbreviations: HbAIC, hemoglobin AIC; DKA, diabetes ketoacidosis; SMBG, self-monitoring of blood sugar; $6 \mathrm{M}$, mean number per six months; day, mean number per day; week, mean number per week; $M$, average number per month.

In pump group we compared the pre-pump and post pump periods, and observed a significant improvement in $\mathrm{HbAlc}$, a lower daily insulin dose, less frequent hypoglycemic episodes and DKA, fewer hospital admissions and higher quality of life and drug satisfaction scores, as shown in Table 3.

We did a subgroup analysis of the pump group that target what we labeled as high risk patients. High risk patients were defined as the patients who had the poorest diabetes control (HbAlc of $10 \%$ and more), worst compliance with SMBG (fewer than four times per day) and inconsistent attendance at diabetic clinic. We found even more remarkable improvement in almost all of the specified parameters. We were unable to evaluate the statistical significance of disease burden because of small sample size. The results from the subgroup analysis are shown in Table 4.

Table 3 Results of pump group, before and after pump installation, $n=40$

\begin{tabular}{llll}
\hline & Pre-pump & $\begin{array}{l}\text { Post- } \\
\text { pump }\end{array}$ & P-value \\
\hline Mean HbAIc (\%) & 9.153 & 7.288 & 0.0001 \\
Insulin (units/day) & 67.85 & 42.13 & 0.0001 \\
Hypoglycemia/week & 3.18 & 1.08 & 0.0001 \\
Severe hypoglycemia/M & 0.63 & 0.15 & 0.006 \\
DKA/6 M & 1.2 & 0.48 & 0.004 \\
Admission/6 M & 1.3 & 0.38 & 0.002 \\
Number of SMBG/day & 2.27 & 3.95 & 0.001 \\
Satisfaction with treatment (\%) & 49.8 & 93.3 & 0 \\
High Burden of the disease (\%) & 93 & 59 & 0
\end{tabular}

Abbreviations: $\mathrm{HbAIC}$, hemoglobin AIC; Week, mean number per week; $M$, average number per month; DKA, diabetes ketoacidosis; $6 \mathrm{M}$, mean number per six months; SMBG, self-monitoring of blood sugar; day, mean number per day.

Table 4 Results of high-risk group before and after the pump installation.

\begin{tabular}{llll}
\hline & Pre-pump & Post-pump & P-value \\
\hline Mean HgAlc (\%) & $1 \mathrm{l} .283$ & 8.25 & 0 \\
Insulin dose (units/day) & 62.25 & 40.58 & $0.00 \mathrm{I}$ \\
Hypoglycemia/week & 3.67 & 0.67 & $0.00 \mathrm{I}$ \\
DKA/6 M & 1.83 & 0.42 & $0.02 \mathrm{I}$ \\
Admission/6 M & 2 & 0.33 & 0.019 \\
Number of SMBG/day & 1.66 & 3.75 & $0.000 \mathrm{I}$ \\
Satisfaction with treatment & 35.72 & 53.27 & $0.00 \mathrm{I}$ \\
$\begin{array}{l}\text { (\%) } \\
\text { High disease burden (\%) }\end{array}$ & 63.6 & 36.4 & $\mathrm{NA}$ \\
\hline
\end{tabular}

Abbreviations: HbAIC, hemoglobin AIC; week, mean number per week; M, average number per month; DKA, diabetes ketoacidosis; 6M, mean number per six months; SMBG, self-monitoring of blood sugar; day, mean number per day; NA, data is not available:

P-Value could not be calculated because of small sample size.

\section{Discussion}

In several observational studies of insulin pump therapy, even with carefully selected candidates, the improvement of Hbalc is usually modest $^{3}$ and is much less remarkable in randomized control trials ${ }^{2-5}$. In this study, we demonstrated a substantial impact of insulin pump therapy on diabetes management and on other aspects of the disease, including the frequency of severe hypoglycemia and quality of life. We think that one of the reasons for this is the supportive services and careful follow-up provided to the patients by our diabetes educators and dietitians. In addition, we believe that most of the patients became more motivated to control their diabetes after beginning insulin pump therapy. This can explain the more significant impact of pump therapy 
on high risk patients although relaxed selection criteria were used to treat them with this modality. Our main goal of using the pump in the high risk group was to ameliorate hyperglycemia and reduce the frequency of DKA. The pump worked very well and not only decreased the number of DKAs and admissions, but also gave all of the expected benefits associated with pump therapy. This was evident in all patients in the pump group and was more pronounced in the high risk group who performed more SMBG and became more consistent in attending the clinic and following instructions from diabetes educators and dietitians (data not provided). Although the pump group showed a remarkable decrease in the frequency of DKA and rate of hospital admission, it was not statistical significant compared to the control group. This finding can possibly be explained by the small number of DKA and sever hypoglycemia events in the control group, these findings are consistent with findings of other studies. ${ }^{4}$ The strengths of our study include the followings: both groups were very well matched with almost all possible confounders and the same selection criteria were used for all pump patients. For its weaknesses, there is a chance that the patients in both the study and control groups were subjected to selection bias. Other weak points are the small sample size and a relatively short duration of insulin pump therapy for some patients in the pump group.

\section{Conclusion}

In our study we showed that insulin pump therapy is remarkably helpful in controlling blood sugar and quality of life in type I diabetes patients and provide many other benefits. We believe that these benefits can be gained even when less strict selection criteria are used as long as the patients receive good supportive services post therapy. We think that more data are needed to validate the criteria for selecting patients for insulin pump therapy.

\section{Acknowledgments}

We thank the patients and families of King Fahad Specialist Hospital for taking part of this study. Special thanks to Mr. Ibrahim El-Hassan Osman, senior biostatistician at King Fahad Specialist Hospital for his great help in analyzing the data.

\section{Funding}

There was no funding support provided to the study.

\section{Authorship}

All named authors meet the International Committee of medical Journal Editors (ICMJE) criteria for authorship for this manuscript, take responsibility for the integrity of the work as a whole, and have given final approval to the version to be published.

\section{Disclosers}

All authors have no conflict of interest.

\section{Compliance with ethics guidelines}

The study was approved by the institutional ethical board at king Fahad Specialist Hospital. All procedures followed were in accordance with the ethical standards of the responsible committee on human experimentation (institutional and national) and with the Helsinki Declaration of 1964, as revised in 2013. Informed consent was obtained from all patients for being included in the study.

\section{References}

1. Deeb LC. Diabetes technology during the past 30 years: a lot of changes and mostly for the better. Diabetes Spectr. 2008;21(2):78.

2. Misso ML, Egberts KJ, Page M, et al. Continuous subcutaneous insulin infusion (CSII) versus multiple insulin injections for type 1 diabetes mellitus. Cochrane Database Syst Rev. 2010;1:CD005103.

3. Cummins E, Royle P, Snaith A, et al. Clinical effectiveness and costeffectiveness of continuous subcutaneous insulin infusion for diabetes: systematic review and economic evaluation. Health Technol Assess. 2010;14(11):1-181.

4. Fatourechi MM, Kudva YC, Murad MH, et al. Clinical review: hypoglycemia with intensive insulin therapy: a systematic review and meta-analyses of randomized trials of continuous subcutaneous insulin infusion versus multiple daily injections. J Clin Endocrinol Metab. 2009;94(3):729-740.

5. Jeitler K, Horvath K, Berghold A, et al. Continuous subcutaneous insulin infusion versus multiple daily insulin injections in patients with diabetes mellitus: systematic review and meta-analysis. Diabetologia. 2008;51(6):941-951.

6. Bott U, Muhlhauser I, Overmann H, et al. Validation of a diabetes-specific quality-of-life scale for patients with type 1 diabetes. Diabetes Care. 1998;21(5):757-769.

7. Grunberger G, Abelseth JM, Bailey TS, et al. Consensus statement by the American association of clinical endocrinologists/American college of endocrinology insulin pump management task force. Endocr Pract. 2014;20(5):463-489. 